\section{New Adhesives for Filter Manufacturing}

$\mathrm{H}^{\mathrm{s}}$ enkel has developed adhesives for the production of filters that have double the temperature resistance of the predecessor product. Macroplast UK 1352 is a polyurethane adhesive that can withstand continuous operating temperatures of up

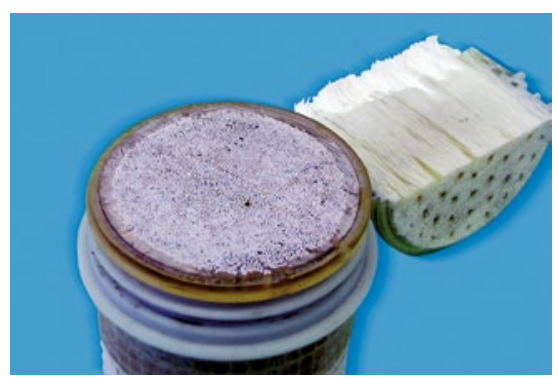
cessed hot, are therefore energy-intensive, and take about one hour to cure.

Equally new in the product range are the two adhesives Macroplast CR 3529 and Macroplast CR 8605, both of which have been approved for use in potable water filto $120{ }^{\circ} \mathrm{C}$. In addition, the innovative adhesive reaches handling strength at room temperature after only 15 minutes. For filter manufacturers, this represents an alternative to traditional epoxy adhesives. These have to be pro-

\section{New Tubular Packaging Solution}

Th he company Poly-clip System has developed a new type of packaging with advantages for opening, dosing and storing pasty products that make it the ideal alternative to conventional tubes and cartridges. This tubular packaging with the brand name ClipTube is a tubular bag with a tamperproof clip and screw closure with a safety system.

The special features of this packaging are simple squeezing without entry of air during the emptying process and a high degree of emptying with less remains of packaged material due to the use of flexible films as the tube body. The pointed shape of the extrusion nozzle ensures exact dosing of the content and its cylindrical shape is very space-saving.

Production takes place on an automatic packaging line, consisting of an automatic transfer sealing and clipping machine with a hydraulic dosing pump or filler and the so-called Cap Bonding System (CBS).

For further information, please contact: Poly-clip System GmbH \& Co. KG, www.polyclip.com

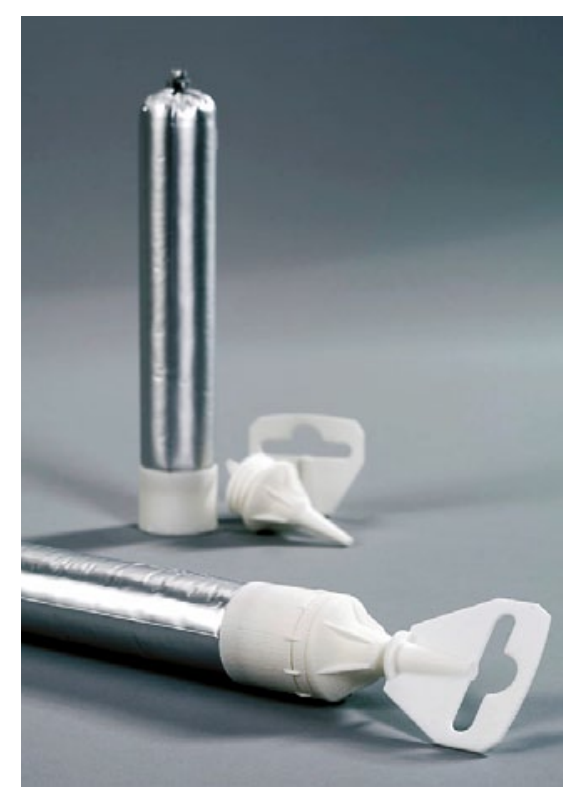

W herever dissimilar materials such as transparent or translucent acrylic, metal, polycarbonate, PVC alloys, ABS, polyurethane or polystyrene need to be bonded, one soIution is to use the adhesive Ultra Light-Weld 3013 from Dymax. This low-viscosity, moisture-resistant adhesive forms high-strength, environmentally resistant adhesive bonds and is suitable, for example, for bonding plastic windows and plastic hous- ings as well as for sealing. It dispenses easily and fluoresces blue under “black" light (365 nm), thus allowing inline quality control using optical scanners. The adhesive cures in seconds on exposure to ultraviolet and visible light, even through UV-blocked plastics.

For further information, please contact: Dymax Europe GmbH, D-65203 Wiesbaden, info_DE@dymax.com, www.dymax.de 